PREPARED FOR THE U.S. DEPARTMENT OF ENERGY, UNDER CONTRACT DE-AC02-76CH03073

PPPL-3828

PPPL-3828

UC-70

Theory and Observations of High Frequency Alfvén

Eigenmodes in Low Aspect Ratio Plasma

by

N.N. Gorelenkov, E. Fredrickson, E. Belova,

C.Z. Cheng, D. Gates, S. Kaye, and R. White

June 2003

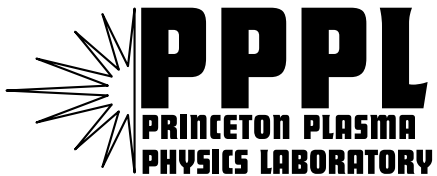

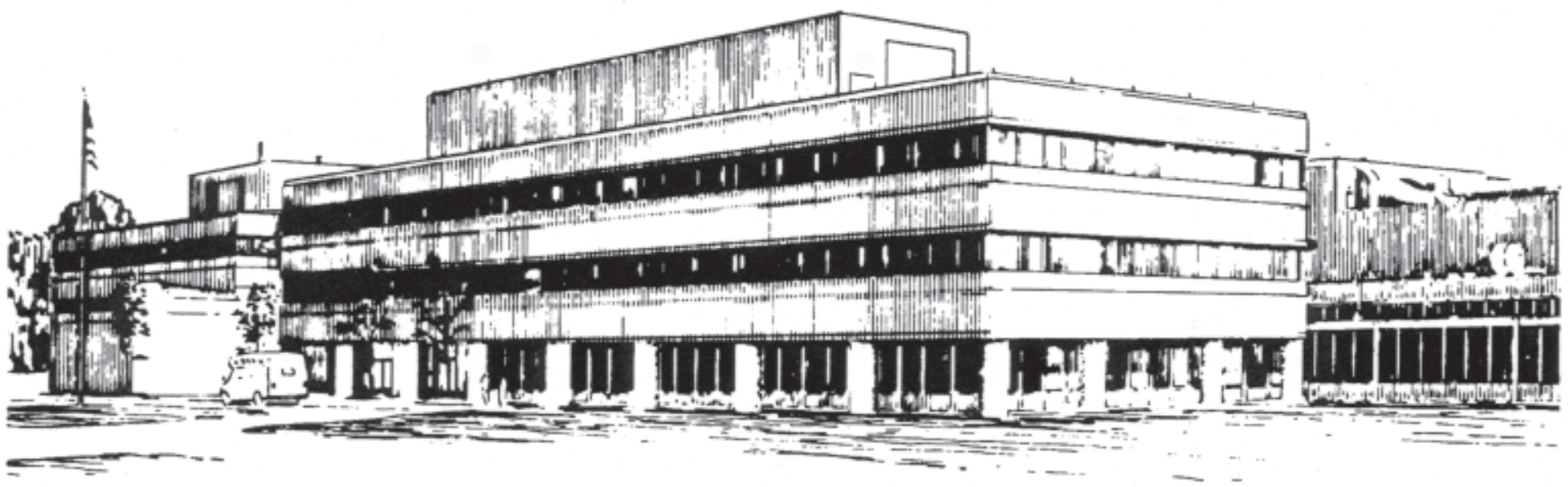

PRINCETON PLASMA PHYSICS LABORATORY PRINCETON UNIVERSITY, PRINCETON, NEW JERSEY 


\section{PPPL Reports Disclaimer}

This report was prepared as an account of work sponsored by an agency of the United States Government. Neither the United States Government nor any agency thereof, nor any of their employees, makes any warranty, express or implied, or assumes any legal liability or responsibility for the accuracy, completeness, or usefulness of any information, apparatus, product, or process disclosed, or represents that its use would not infringe privately owned rights. Reference herein to any specific commercial product, process, or service by trade name, trademark, manufacturer, or otherwise, does not necessarily constitute or imply its endorsement, recommendation, or favoring by the United States Government or any agency thereof. The views and opinions of authors expressed herein do not necessarily state or reflect those of the United States Government or any agency thereof.

\section{Availability}

This report is posted on the U.S. Department of Energy's Princeton Plasma Physics Laboratory Publications and Reports web site in Fiscal Year 2003. The home page for PPPL Reports and Publications is: http://www.pppl.gov/pub_report/

DOE and DOE Contractors can obtain copies of this report from:

U.S. Department of Energy

Office of Scientific and Technical Information

DOE Technical Information Services (DTIS)

P.O. Box 62

Oak Ridge, TN 37831

Telephone: (865) 576-8401

Fax: (865) 576-5728

Email: reports@adonis.osti.gov

This report is available to the general public from:

National Technical Information Service

U.S. Department of Commerce

5285 Port Royal Road

Springfield, VA 22161

Telephone: $1-800-553-6847$ or

(703) $605-6000$

Fax: (703) 321-8547

Internet: http://www.ntis.gov/ordering.htm 
2nd July 2003

\title{
Theory and Observations of High Frequency Alfvén Eigenmodes in Low Aspect Ratio Plasma.*
}

\author{
N. N. Gorelenkov 1), E. Fredrickson 1), E. Belova 1), C. Z. Cheng 1) \\ D. Gates 1), S. Kaye 1), R. White 1)
}

1) Princeton Plasma Physics Laboratory, P.O. Box 451, Princeton, NJ, USA 08543-0451

e-mail contact of main author: ngorelen@pppl.gov

\begin{abstract}
New observations of sub-cyclotron frequency instability in low aspect ratio plasma in National Spherical Torus experiments (NSTX) are reported. The frequencies of observed instabilities correlate with the characteristic Alfvén velocity of the plasma. A theory of localized Compressional Alfvén Eigenmodes (CAE) and Global shear Alfvén Eigenmodes (GAE) in low aspect ratio plasma is presented to explain the observed high frequency instabilities. CAE's/GAE's are driven by the velocity space gradient of energetic super-Alfvénic beam ions via Doppler shifted cyclotron resonances. One of the main damping mechanisms of GAE's, the continuum damping, is treated perturbatively within the framework of ideal MHD. Properties of these cyclotron instabilities ions are presented.
\end{abstract}

PACS numbers: 52.35.Bj, 52.35.Qz, 52.40.Mj, 52.50.Gj, 52.55.Fa.

*This work supported by DoE contract No. DE-AC02-76CH03073 


\section{Introduction}

Magnetic field activity measured by edge Mirnov coils during the NBI injection in NSTX shows a broad and complicated frequency spectrum of coherent modes between $400 \mathrm{kHz}$ and up to $2.5 \mathrm{MHz}$, with the fundamental cyclotron frequency of background deuterium ions $f_{c D}=\omega_{c D} / 2 \pi=2.3 \mathrm{MHz}$, calculated using the vacuum magnetic field at the geometrical axis of the plasma $B_{g 0}=0.3 T[1,2,3]$. The instability frequency spectrum has discrete peaks as shown in Figure 1. Spectrum peak frequencies correlate with the characteristic Alfvén velocity as magnetic field and plasma density are varied. The NSTX is a low aspect ratio toroidal device with major and minor radii $R_{g 0}=$ $0.85 m$ and $a=0.65 m$ respectively. In NSTX the Alfvén speed is low compared to the injection velocity of $E_{b 0} \simeq 80 \mathrm{keV} \mathrm{NBI}$ deuterium ions, with $v_{A} / v_{b 0} \simeq 1 / 4$. The power available to sustain these modes may be on the order of the total auxiliary heating power, which is much larger than in conventional tokamaks and thus much stronger Alfvén type instabilities are expected in NSTX. The excitation is sensitive to the NBI injection angle. Particle losses were not seen during these instabilities.

The sub-cyclotron frequency instabilities under consideration are shear Alfvén and compressional Alfvén (or magnetosonic) types of plasma oscillations. For the purpose of application to the low aspect ratio plasma of Spherical Tokamaks (ST) we developed a new theory of radially and poloidaly localized Compressional Alfvén Eigenmodes (CAE) [3, 4]. Initially observed instabilities were identified as CAEs driven by energetic beam ions since the predicted CAE frequency spectra were in agreement with the experiments, in which the magnetic signal peaks evolve parallel to each other. CAEs are localized radially at the plasma edge and poloidaly on the low magnetic field side

of the torus. CAE frequencies are determined primarily by the Alfvén frequency at the mode location and the poloidal wave vector: $\omega_{C A E}=v_{A} m / r$, where $m$ is the poloidal mode number, and $r$ is the minor radius.

In new observations such as shown in Fig. 1 the spectrum peak lines intersect one another which indicates more complicated dispersion than reported earlier for CAEs. We suggest that such modes are GAE's [5]. GAEs were found to be unstable in a nonlinear Hybrid MHD (HYM) code [6, 7] modified for the ST geometry (to be reported elsewhere). In conventional tokamaks GAE's were shown to be stable against the fast particle pressure driven instability as a result of strong continuum damping. This is due to the toroidal coupling to the kinetic mode at the edge [11]. In this report 
we summarize the theory and observations of CAEs and GAE's in NSTX and derive the perturbative expression of GAE continuum damping within the ideal MHD.

\section{Compressional Alfvén Eigenmodes}

For a typical low aspect ratio plasma of NSTX the dispersion of CAEs can be presented in the form [4]

$$
\begin{aligned}
\omega_{m s n}^{2} & \simeq \frac{v_{A 00}^{2}}{r_{0}^{2}}\left\{\frac{4(m+1 / 2)^{2}}{\kappa^{2}}\left(\epsilon_{0}-\alpha_{0}\right)+\frac{\kappa^{4}-1}{\kappa^{4}} \frac{m^{2}+m+3 / 2}{2}\right. \\
& \left.+\frac{2(2 s+1)(2 m+1)}{\kappa} \sqrt{\frac{\left(\epsilon_{0}-\alpha_{0}\right)(1+\sigma)}{2 \sigma}}+n^{2}\left[\frac{q^{2}\left(r_{0}\right)}{\kappa^{2}}+\frac{R_{0}^{2}+4 r_{0}^{2}}{4 R_{00}^{2}}\right]\right\} .
\end{aligned}
$$

where $m, n$, and $s$ are the poloidal, toroidal and radial wave numbers, $\alpha_{0} \simeq B_{\theta}^{2} / 2 B_{\varphi}^{2}$ measures the weakening of the magnetic field well due to the poloidal field, $r_{0}=$ $a / \sqrt{1+\sigma}, \kappa$ is the ellipticity, where the plasma density profile was chosen as $n_{e}=$ $n_{e 0}\left(1-r^{2} / a^{2}\right)^{\sigma}$. Double subscript refers to the low field side point at $r=r_{0}$. To the lowest order in $m \gg 1$ the mode is localized at the low field side within poloidal width $\Theta=1 / \sqrt{\epsilon_{0}-\alpha_{0}}$. Radially the CAE is localized within the domain $\Delta^{2} / r_{0}^{2}=$

$\kappa \sqrt{2 \sigma /(1+\sigma)\left(\epsilon_{0}-\alpha_{0}\right)} /(2 m+1)$, and the mode structure is given by solutions in the form

$$
E=e \phi_{m}(\sqrt{2} \theta / \Theta) \phi_{s}\left(\sqrt{2}\left(r-r_{0}\right) / \Delta\right)
$$

where $\phi_{s}(x)=e^{-x^{2} / 2} H_{s}(x) / \sqrt{n ! 2^{s} \sqrt{\pi}}$ and $H_{s}$ are the $s-t h$ order Chebyshev-Hermit functions and polynomials. To avoid the damping on the Alfvén continuum at the edge CAEs must have low $k_{\|}$at the edge and thus low $n$ 's, so that $k$ and the dispersion are primarily determined by the poloidal mode numbers. Note that $m$ in Eq.(2) is a quantum number and becomes the cylindrical poloidal mode number in the limit of high $m$ 's and high aspect ratio[4, 3]. Spacing between the discrete peaks of observed CAE frequency spectra is in agreement with the theoretically obtained dispersion expression. In discharges with pure CAEs the radial mode number corresponds to the frequency spacing on the order of $\Delta f_{s} \simeq 1 M H z$ and is responsible for two bands of $\mathrm{CAE}$ peaks at around $f \simeq 0.9 \mathrm{MHz}$ and $f \simeq 1.8 \mathrm{MHz}$. Within each band the peaks are typically separated by $\Delta f_{m} \simeq 100-150 \mathrm{kHz}$ corresponding to neighboring poloidal mode numbers $m$ and $m+1$. Toroidal mode numbers produce the finest splitting of each frequency peak by $\Delta f_{n} \simeq 10-20 k H z$.. These features were observed 
in experiments.

\section{Global shear Alfvén Eigenmodes}

GAE's are formed just below the minimum of the Alfvén continuum $\omega \simeq \pm \omega_{\text {Amin }}=$ $\left(k_{\|}(r) v_{A}(r)\right)_{\min }[5]$. Here and below subscript min means that the value is taken at the minimum $\omega_{A m i n}$. The GAE eigenfrequency is slightly shifted downward from $\omega_{\text {Amin }}$, and the shift depends on the $q$ and density profiles. GAE is localized radially near the minimum $\omega_{\text {Amin }}$ and is dominated by one poloidal harmonic $m$. With typically flat $q$ profile the Alfvén continuum has a minimum at the plasma center, so that $\omega \simeq \pm v_{A 0}\left(m / q_{0}-n\right) / R_{a x}$, where $R_{a x}$ is the major radius of the magnetic axis. One can see that if $q$ is evolving in time eigenfrequencies of GAE's with different combinations of $(m, n)$ will have different time dependencies. This can be seen from figure 1, where the instability peaks intersect. In this figure we plotted several simplified GAE eigenfrequencies $\omega \simeq-\omega_{\text {Amin }}$. The choice of the sign is made to match the calculated frequency with the observed and to satisfy the resonance condition of co-injected beam ions with CAE/GAE's (see next section). Some discrepancy with the measured frequencies is due to the uncertainties in the measurements of the plasma parameters and in the reconstruction of the equilibrium with the EFIT equilibrium code. We calculate the AE continuum and one of the GAE's using the ideal MHD code NOVA [8] as shown in figure 2. The frequency of this mode in the laboratory frame is $f=\left(1.18+n f_{\text {rot }}\right) M H z$, where $f_{\text {rot }}=11 k H z$ accounts for the plasma rotation. This frequency closely (within 5\%) matches the observed frequency. The frequency separation between the different modes is primarily due to the changes in $n$ or $m$, so that $\Delta f=f_{m, n+1}-f_{m, n}=v_{A 0} / R-f_{\text {rot }}=175 k \mathrm{~Hz}$ or $\Delta f=f_{m+1, n}-f_{m, n}=v_{A 0} / q_{0} R=150 k H z$.

The problem of finding the eigenmode structure needs numerical solution for the general plasma equilibrium and was studied in Ref. [5]. A proper eigenmode structure is necessary to determine one of the main contribution to GAE damping, which is the continuum damping due to the interaction with the $m+1$ harmonic Alfvén continuum on the tail of the eigenfunction [11]. GAE approximate solution and dispersion were found analytically in Refs. $[9,10]$ in the case when the Alfvén continuum minimum is at $r=r_{\min } \neq 0$. In spherical tokamak conditions with high beta (as one can see from the example in Fig. 2) it is more practical to consider a case of $r_{\min }=0$, which 
we address in the remainder of this section. The shift of the minimum of the Alfvén continuum to the plasma center is caused by such effects as strong Shafranov shift and low aspect ratio.

\subsection{GAE mode structure}

To calculate the GAE eigenfunction we consider the ideal MHD equation for the shear Alfvén plasma oscillations in the cylinder for a mode with $(m, n)$ wave numbers $[9,12]$ :

$$
\frac{d}{d r}\left[u \frac{d}{d r} E_{m}\right]-r^{-2}\left(m^{2}-1\right) u E_{m}+\frac{d}{a d r}\left(\frac{\omega^{2}}{v_{A}^{2}}\right) r^{2} E_{m}+\frac{d}{a d r}\left[r^{3} \hat{\epsilon} \frac{\omega^{2}}{v_{A}^{2}} \frac{d}{d r} E_{m+1}\right]=0
$$

where $u=r^{3}\left(\omega^{2} / v_{A}^{2}-k_{\| m}^{2}\right) / a, E_{m}=\Phi_{m} / r, \Phi_{m}$ is the $m$ th harmonic of the perturbed electrostatic potential, and the coupling parameter of the $m$ and $m+1$ harmonics, $\hat{\epsilon}=$ $5 r / 2 R_{0}$, is obtained in high aspect ratio and flat current profile limit. Approximately $\omega / \omega_{c}<1$ corrections can be accounted for by re-normalizing the frequency of the mode as follows $\omega^{2} \rightarrow \omega^{2} /\left(1-\omega^{2} / \omega_{c}^{2}\right)$, but will be neglected in this paper for the sake of simplicity. We restrict analysis to the case of interaction of only two poloidal harmonics, so that the other equation required for the system is similar to Eq.(3) with the subscript substitution $m \rightarrow m+1$ and $m+1 \rightarrow m$.

We will solve this system by the perturbation technique assuming $\hat{\epsilon} \ll 1$ and $m \gg 1$, so that unperturbed solution is obtained when two harmonics are considered decoupled and $E_{m+1}^{0}(r)=0$. Note that since the GAE is expected to be localized near the center [11] the quadratic form allows inclusion of weakly coupled $m+1$ harmonic perturbatively (see below section 3.2) and assumptions we use seem adequate. For the zero order $m$ th harmonic electric field we introduce the new function $\xi=E_{m}^{0} \sqrt{u}$. Then we obtain an eigenmode equation

$$
\frac{d^{2}}{d x^{2}} \xi-V \xi=0
$$

with the potential in the form $V(x)=\left(m^{2}-1\right) / x^{2}+(\ln u)^{\prime \prime} / 2+(\ln u)^{\prime 2} / 4-r^{2} \omega^{2}(\ln n)^{\prime} / u v_{A}^{2}$, $f^{\prime} \equiv d f / d x, x=r / a$. Let us consider the following profile of the Alfvén frequency continuum $\omega_{A}^{2}=\omega_{A 0}^{2}+D x^{\delta}, D>0$, and density profile as specified above. Note that such form of the cylindrical continuum is more convenient to fit to the realistic Alfvén 
continuum. Then the potential equals

$$
\begin{aligned}
V(x) & =\frac{m^{2}-1 / 4}{x^{2}}-\frac{\sigma\left(1+x^{2}\right)}{\left(1-x^{2}\right)^{2}}-\frac{x^{\delta-2}\left((\delta-1) g+x^{\delta}\right)}{\left(g-x^{\delta}\right)^{2}}+\frac{1}{4}\left[\frac{3}{x}-\frac{2 \sigma x}{1-x^{2}}-\frac{x^{\delta-1} \delta}{g-x^{\delta}}\right]^{2} \\
& -\frac{9}{4 x^{2}}+\frac{2 \sigma\left(g+\omega_{A 0}^{2} / D\right)}{\left(g-x^{\delta}\right)\left(1-x^{2}\right)}
\end{aligned}
$$

where $g=\left(\omega^{2}-\omega_{A 0}^{2}\right) / D$. One can show that this potential is shaped near the center mostly due to the first and the last terms in Eq.(5) $V(x) \simeq\left(m^{2}-1 / 4\right) / x^{2}+$ $2 \sigma \omega_{A 0}^{2} / D\left(g-x^{\delta}\right)+C$, where $C$ equals the rest of terms in Eq. $(5)$ evaluated at the minimum, $C \simeq$ const $=\left[(\ln u)^{\prime \prime} / 2+(\ln u)^{\prime 2} / 4-9 / 4 x^{2}\right]_{\mid x=x_{0}}$. The minor radius of the minimum of the potential, $x_{0}$, is determined by the equation $V^{\prime}\left(g, x_{0}\right)=0$, which is transcendental in general: $g-x_{0}^{\delta}=x_{0}^{\delta / 2+1} \sqrt{\delta \sigma \omega_{A 0}^{2} / D\left(m^{2}-1 / 4\right)}$. This equation also determines the condition for the potential well minimum to exist inside $0<x_{0}<1$. Analytical solution of this equation is allowed for $\delta=2$ : $x_{0}^{2}=$ $g /\left(1-\sqrt{\delta \sigma \omega_{A 0}^{2} / D\left(m^{2}-1 / 4\right)}\right)$. One can see that low-m GAE's are easier to satisfy this condition for a given $\omega_{A}(r)$ dependence. Numerically one can also show that at higher $\delta$, i.e. at more flat continuum profile, the condition for the existence of the potential well minimum, which is the necessary condition for GAE localization, is easier to satisfy.

Within the framework of WKB the quantization condition for GAE localization is

$$
\int_{x_{1}}^{x_{2}} \sqrt{-V} d x=\pi \frac{1+s}{2}
$$

where $s$ is the radial mode number and $x_{1,2}$ are the turning points $\left(V\left(x_{1}\right)=V\left(x_{2}\right)=0\right)$ bounding the region where $V<0$. The solution in the WKB limit can be given by $\xi_{s}(x)=\exp \left\{\left[1-2 \eta\left(x-x_{2}\right)\right] \int_{x_{1}}^{x} \sqrt{V} d x\right\}$, where $\eta(x)$ is the Heaviside step function. For $\delta=2$, however, and $m \gg 1$ there is no solution for $x_{2}$, which means that if the continuum is not flat enough near the center the GAE can propagate to the edge of the plasma.

If $\delta>2$ we find the solution approximately and can write $x_{0} \simeq x_{2} \simeq(-g)^{1 / \delta}$ and approximate $V(x) \simeq\left(m^{2}-1 / 4\right) / x^{2}$ if $x>x_{2}$ and $V(x) \simeq\left(m^{2}-1 / 4\right) / x^{2}+$ $2 \sigma \omega_{A 0}^{2} / D g+C$ if $x<x_{2}$. In such a case the solution of the eigenmode equation for $x<x_{2}$ can be expressed through the Bessel functions of order $m$ :

$$
\xi(x)=e_{0} \sqrt{x} J_{m}\left(\left(-2 \sigma \omega_{A 0}^{2} / D g-C\right)^{1 / 2} x\right)
$$


where we imposed the boundary condition at the center $\xi(0)=0$. Outside of the potential well, $x>x_{2}$, the solution is $\xi(x) \sim e^{1} x^{1 / 2-m}$. The dispersion relation is obtained by matching two parts of the solution and their first derivatives at $x=x_{2}$. We obtain $e_{1}=e_{0} J_{m} x_{2}^{m}$ and the dispersion equation $J_{m-1}\left(\left(-2 \sigma \omega_{A 0}^{2} / D g-C\right)^{1 / 2}(-g)^{1 / \delta}\right)=0$ or

$$
\left(-2 \sigma \omega_{A 0}^{2} / D g-C\right)^{1 / 2}(-g)^{1 / \delta}=j_{m-1, s+1}
$$

where $j_{m-1, s+1}$ is the standard notation for the zeros of the Bessel function. The first zero, corresponding to the first radial eigenmode at $s=0$ in the high $m$ limit is $j_{m-1,1} \simeq m+1.86 m^{1 / 3}+m^{-1 / 3}$. Thus the eigenmode structure of the GAE with $s$ th radial number is determined by

$$
\xi_{s}(x)= \begin{cases}e_{0} \sqrt{x} J_{m}\left(j_{m-1, s+1} x / x_{2}\right), & x<x_{2} \\ e_{0} J_{m}\left(j_{m-1, s+1}\right) x_{2}^{m} x^{1 / 2-m}, & x>x_{2},\end{cases}
$$

where $x_{2}$ is to be found by solving Eq.(8).

\subsection{GAE continuum damping}

To calculate the continuum damping we will follow the perturbative procedure similar to one outlined in Refs.[12,13] in application to toroidicity induced Alfvén eigenmodes. For that we construct the quadratic form by multiplying Eq.(3) by $E_{m}^{*}$ and integrating it over the minor radius. To zero order in $\hat{\epsilon}$ the quadratic form is $G\left(\omega_{0}\right) \equiv \int d r\left[a u\left(E_{m}^{0 / 2}+\frac{m^{2}-1}{r^{2}} E_{m}^{02}\right)-\frac{d}{d r}\left(\frac{\omega_{0}^{2}}{v_{A}^{2}}\right) r^{2} E_{m}^{02}\right]=0$, where $E_{m}^{0}=\xi_{s} / \sqrt{u}$. The first order correction to the quadratic form includes the $m+1$ th harmonic. One can see from Eq.(3) written for $m+1$ harmonic that $u$ vanishes at the $m+1$ th harmonic continuum, $r=r_{s}$, so that $E_{m+1}^{1}$ has a discontinuity, but its contribution to the quadratic form is small $O(\hat{\epsilon})$. In the corresponding equation for the $m+1$ harmonic (see Eq.(3)) we will keep only the first and last terms near $r_{s}$. In the limit of high $m$ one can estimate $x_{s}^{\delta} \simeq 2 \omega_{A 0}^{2} / D R q k_{\| m}(0)\left(1-x_{s}^{2}\right)^{\sigma / 2} \simeq 2 \omega_{A 0}^{2} / D R q_{0} k_{\| m}(0)$. To treat the singularity near $r_{s}$ we apply the causality condition that the frequency is formally in the upper half-plane, $\omega_{0}+i \nu, \nu>0$ and take the limit of $\nu \rightarrow 0$, so that near the singular point $r_{s}$

$$
\frac{d}{d r}\left[r^{3}\left((\omega+i \nu)^{2} / v_{A}^{2}-k_{\| m+1}^{2}\right) \frac{d}{d r} E_{m+1}^{1}\right]+\frac{d}{d r}\left[r^{3} \hat{\epsilon} \frac{\omega_{0}^{2}}{v_{A}^{2}} \frac{d}{d r} E_{m}^{0}\right]=0 .
$$


In this case the quadratic form can be again obtained from Eq.(3) similarly to the zero order limit and results in the equation $d G / d \omega_{0} \delta \omega=-\int d r r^{3}\left(E_{m}^{0}\right)^{\prime}\left(E_{m+1}^{1}\right)^{\prime} \hat{\epsilon}\left(\omega_{0}^{2} / v_{A}^{2}\right)$, where we denoted the first order correction to the eigenfrequency as $\delta \omega$. Integrating Eq.(10) by parts and keeping only the imaginary part of the frequency correction we can write

$$
\Im \delta \omega\left[\frac{d G}{d \omega_{0}}\right]=\lim _{\nu, \delta \rightarrow 0} \Im \int_{r_{s}-\delta}^{r_{s}+\delta} d r \frac{r^{3} \hat{\epsilon}^{2}\left(E_{m}^{0}\right)^{\prime 2} \omega_{0}^{4} v_{A}^{-2}}{\left(\omega_{0}+i \nu\right)^{2}-v_{A}^{2} k_{\| m+1}^{2}}=-\left.\pi \frac{r_{s}^{3} \hat{\epsilon}^{2}\left(E_{m}^{0}\right)^{\prime 2} \omega_{0}^{3}\left|\omega_{0}\right|}{v_{A}^{2}\left|\partial v_{A}^{2} k_{\| m+1}^{2} / \partial r\right|}\right|_{r_{s}} .
$$

This is the general expression for GAE continuum damping, which we will simplify in the following in the high- $m$ limit and using the above parametrization. We then find $\left|\partial v_{A}^{2} k_{\| m+1}^{2} / \partial r\right| \simeq x_{s}^{\delta-1} D \delta / a$ and $d G / d \omega_{0} \simeq 4 e^{02} \omega_{0} m^{2} I_{m, s} / a\left(\omega_{A 0}^{2}-\omega_{0}^{2}\right)$, where $I_{m, s}=\int_{0}^{x_{2}} d x J_{m}^{2} / x$. Thus with the help of Eq.(9) we obtain

$$
\Im \frac{\delta \omega}{\left|\omega_{0}\right|}=-\frac{\pi}{4} \frac{\hat{\epsilon}_{s}^{2} \omega_{0}^{2}}{\delta D} \frac{x_{2}^{2 m+\delta}}{x_{s}^{2 m+2 \delta}} \frac{J_{m}^{2}\left(j_{m-1, s+1}\right)}{I_{m, s}} .
$$

It is clear that this contribution is always stabilizing. For practical purposes we compute numerically $J_{m}^{2}\left(j_{m-1, s+1}\right) / I_{m, s} \simeq 1.95 m^{2 / 3} /\left(m^{2 / 3}+5 / 3\right)$ for $1<m<100$ with better than $1 \%$ accuracy. One can notice that as long as $x_{2}<x_{s}$, i.e. the singularity is outside the mode localization, the high- $m$ modes are slightly damped, $\Im \delta \omega /\left|\omega_{0}\right| \sim\left(x_{2} / x_{s}\right)^{2 m+\delta}$.

Let us consider an example of the continuum damping of $m=-4$ GAE in NSTX, $R_{0} / a=1.3$, and fix $\sigma=0.5, \delta=4, \omega_{0}^{2} / D=1$. The dispersion equation for $s=0$ (Eq.(8)) gives $g=-0.48 \times 10^{-3}$, and we also obtain $x_{2}=0.148, x_{s}=0.7$. The damping rate from Eq.(12) gives the very low value $\Im \delta \omega /\left|\omega_{0}\right|=-0.12 \times 10^{-7}$. The maximum continuum damping $\left(\Im \delta \omega /\left|\omega_{0}\right|\right)_{\max } \sim 1$ is achieved when the singular surface is within the mode localization, that is $x_{2}=x_{s}$. In this case our perturbative technique is not valid. Low value of the damping we calculated is due to the chosen large $\delta$, which makes the mode localized near the center and separated from the $m+1$ continuum. Low poloidal mode number GAE $m \leq 2$ should suffer stronger damping from the continuum due to a global structure extended enough to stabilize the beam ion drive. This wlll be estimated in the next section. Stronger damping is expected for non-flat Alfvén continuum such as $\delta=2$. For practical purposes Eq.(11) has to be applied with the substitution of the numerical eigenmode structure. 


\section{CAE/GAE cyclotron excitation by beam ions}

Since CAE's are localized at the edge and GAE's are localized at the center we consider a WKB approximation. A more general system of equations than considered above for the perturbed electric field in a homogeneous plasma is

$$
\begin{array}{r}
\left(k_{\perp}^{2}-F\right) E_{1}-H E_{2}=0 \\
H E_{1}-F E_{2}=0
\end{array}
$$

where $k_{\perp}, k_{\|}$are the perpendicular and parallel wavevectors, $F=\omega^{2} \epsilon_{11} / c^{2}-k_{\|}^{2}, H=$ $\omega^{2} \epsilon_{12} / c^{2}$, dielectric tensor elements are $\epsilon_{11}=\epsilon_{22}=\omega_{p i}^{2} /\left(\omega_{c i}^{2}-\omega^{2}\right), \epsilon_{12}=-\epsilon_{21}=$ $i \omega \omega_{p i}^{2} / \omega_{c i}\left(\omega_{c i}^{2}-\omega^{2}\right)$ and we ignored the parallel electric field. Here we have chosen direction 1 to be perpendicular to the vector $\mathbf{k}_{\perp}$, and to the equilibrium magnetic field. The dispersion equation for both branches is $F\left(k_{\perp}^{2}-F\right)-H^{2}=0$. If $|\omega| \ll \omega_{c i}$ the two branches are decoupled and satisfy two equations: $F=0$ for shear Alfvén and $k_{\perp}^{2}-F=0$ for compressional Alfvén oscillations. Equation (14) gives the electric field polarization in case of $|\omega|<\omega_{c i}, E_{1}=E_{2} H / k_{\perp} \ll E_{2}$ for GAE's and in the limit of $k_{\|} \ll k, E_{1}=E_{2} F / H \simeq-i E_{2} \omega_{c i} / \omega$ for CAEs.

The perturbation theory expression for the growth rate of the cyclotron instability driven by fast beam ions is described in Ref. [14] and includes particle drift motion which strongly modifies the resonant interaction with the eigenmode:

$$
\gamma_{b}=-\omega \int d \mathbf{r} E^{*} \Im \epsilon_{b}^{A} E / 2 \int d \mathbf{r} \mathbf{E}^{*} \epsilon \mathbf{E},
$$

where subscript $b$ refers to the beam ions, and $\epsilon_{b}^{A}$ is the anti-hermitian part of beam ion dielectric tensor. For CAEs we obtain $\mathbf{E}^{*} \epsilon \mathbf{E}=\left|E_{1}\right|^{2} \omega_{p i}^{2} / \omega_{c i}^{2}$ and for GAE's $\mathbf{E}^{*} \epsilon \mathbf{E}=$ $\left|E_{2}\right|^{2} \epsilon_{11}\left(1+2 \omega^{4} \epsilon_{11} / \omega_{c i}^{2} k_{\perp}^{2} c^{2}\right)$. In the WKB approximation the contribution of the anti-hermitian part of beam ion dielectric tensor is given by the formula

$$
\int d \mathbf{r} \mathbf{E}^{*} \Im \epsilon_{b}^{A} \mathbf{E}=-\frac{2 \pi Z_{b}^{2} e^{2}}{\omega m_{b} q R} \sum_{l} \int d \mathbf{r} d \mathcal{E} d \mathcal{E}_{\perp} \delta\left(\theta-\theta_{r e s}\right) I \mathbf{G}_{l}^{* *} \mathbf{E}^{*} \mathbf{G}_{l} \mathbf{E}\left[\frac{\partial}{\partial \mathcal{E}}+\frac{l \omega_{c b}}{\omega} \frac{\partial}{\partial \mathcal{E}_{\perp}}\right] f_{b}
$$

where $q$ is the safety factor, $\mathcal{E}=v^{2} / 2$, and $I$ is the resonance factor, $G_{l}=\left\{-i v_{\perp} \partial J_{l} / \partial z ; v_{\perp} J_{l} / z\right\}$, $G_{l}^{\prime}=\left\{-i v_{\perp} \partial J_{l} / \partial z ; v_{\perp} l J_{l} / z\right\}$ and $\theta_{\text {res }}$ is determined by the resonance condition $\omega-$ $l \omega_{c b}-k_{\|} v_{\|}=0$. From Eq.(16) one can show that the strongest instability is the one 
with $l \neq 0$, for which we restrict our analysis in this paper. In such a case the variation of the phase in the wave particle interaction comes from the $l \omega_{c b}$ term in the resonance condition. The first term in the square brackets of Eq.(16) contributes to the damping or to the drive depending on the distribution function anisotropy. For estimates it is useful to take the limit $\omega^{2} / \omega_{c b}^{2} \ll 1$ and introduce the function $g$ according to the following. For $\mathrm{CAE} \mathbf{G}_{l}^{*} \mathbf{E}^{*} \mathbf{G}_{l} \mathbf{E} \simeq E_{1}^{2} v_{\perp}^{2}\left(J_{0}-J_{2}\right)^{2} / 4 \equiv g v_{\perp}^{2} E_{1}^{2} / 4$ and for $\mathrm{GAE}$ $\mathbf{G}_{l}^{*} \mathbf{E}^{*} \mathbf{G}_{l} \mathbf{E} \simeq E_{1}^{2} v_{\perp}^{2}\left(J_{0}+J_{2}\right)^{2} / 4 \equiv g v_{\perp}^{2} E_{2}^{2} / 4$. In the case of co-injection we have to chose $l=-1$ and $\omega<0$. Then the expression for the beam ion anti-hermitian part of the dielectric tensor is

$$
\int d \mathbf{r} \mathbf{E}^{*} \Im \epsilon_{b}^{A} \mathbf{E}=\frac{\pi \omega_{p b}^{2} l \omega_{c b}}{\omega^{2}} \int d \mathbf{r} d v_{\|} d v_{\perp} E_{i}^{2}\left[\frac{\partial\left(v_{\perp}^{2} g\right)}{\partial v_{\perp}}-\frac{v_{\perp}^{3} g \omega}{v^{2} l \omega_{c b}}\left(\frac{v \partial}{\partial v}-\frac{\lambda \partial}{\partial \lambda}\right)\right] f_{b} \delta\left(\omega-l \omega_{c b}-k_{\|} v_{\|}\right)
$$

where $\lambda \equiv v_{\perp} / v$. The NBI injection in NSTX is tangential. Typically the distribution function can be separated into two parts: almost tangentially confined passing with narrow width in pitch angle $f_{b p}=3 B^{2} \beta_{b}(1-\eta) e^{-\lambda / \delta \lambda_{p}} / v^{3} \delta \lambda_{p}^{2} 2^{3} \pi \mathcal{E}_{b 0}$ and trapped bump-on-tail in $v_{\perp}$ direction, which we assume as Gaussian with narrow width $f_{b t}=3 B^{2} \beta_{b} \eta \sqrt{1-\lambda_{0}^{2}} e^{-\left(\lambda-\lambda_{0}\right)^{2} / \delta \lambda_{t}^{2}} / v^{3} \delta \lambda_{t} \lambda_{0} 2^{3} \pi^{3 / 2} E_{b 0}$, where $\eta$ gives a fraction of trapped ions. For the dense plasma $\eta$ can be close to one, while for the low density plasma it is much smaller than 1.

The integrand determines conditions for the instability. In particular we show the dependence of the driving term in the integrand of Eq.(17), i.e. the quantity $\left(k_{\perp} / \omega_{c b}\right) \partial\left(v_{\perp}^{2} g\right) / \partial v_{\perp}$, in figure 3 . It can be shown that the contribution from passing particles is small as $O\left(\delta v_{\perp b p} / v_{\perp b 0}\right)$ in comparison with the trapped ion contribution, but may be large if passing particles are dominant, i.e. when $\eta \ll 1$. Comparing the integrand of Eq.(17) from figure 3 we conclude that at the low end for the instability one should have $1<k_{\perp} \rho_{\perp b}<2$ or $1<\left(l \omega / \omega_{c b}\right)\left(v_{\perp b 0} / v_{A}\right)<2$ for CAEs and $2<$ $\left(l \omega / \omega_{c b}\right)\left(v_{\perp b 0} / v_{A}\right)\left(k_{\perp} / k_{\|}\right)<4$ for GAE's. Both conditions can be met in NSTX. Note that the finite width of the distribution function in $v_{\perp}$ (denote it $\delta v_{\perp b t}$ ) is stabilizing. The requirement for such stabilization can be obtained from figure 3 so that for CAEs we need $\delta v_{\perp b t}>-v_{A} \omega_{c b} / \omega$, and for GAE's $\delta v_{\perp b t}>-2 v_{A} \omega_{c b} / \omega$. If the distribution function is narrower then the assumption of narrow pitch angle width of the trapped particle distribution function we use is reasonable. After some algebra we arrive at 
the expression for GAE growth rate:

$$
\frac{\gamma_{b G A E}}{\omega}=\frac{-T_{i} \beta_{b}}{E_{b 0} \beta_{i}} \frac{3 \pi \omega_{c b}\left(l \omega_{c i}+\omega\right)}{2 \omega^{2}}\left\{(1-\eta)\left[2-6 \delta \lambda_{p}^{2} \frac{\omega}{l \omega_{c b}}\right]+\eta\left[\frac{\partial\left(v_{\perp}^{2} g_{t}\right)}{v_{\perp} \partial v_{\perp}}-\frac{\omega \lambda_{0}^{2} g_{t}}{l \omega_{c b}}\right]\right\},
$$

where $g_{t}=g\left(\lambda=\lambda_{0}\right)$. For CAEs the growth rate expression is similar $\gamma_{b C A E} \simeq$ $\gamma_{b G A E}$, whereas $g_{t}$ function is to be taken according to $\mathrm{CAE}$ resonance condition. This expression gives the growth rates on the order of $\gamma_{b} /|\omega| \simeq n_{b t} / n_{i} \leq 1 \%$, where $n_{b t}$ is the characteristic density of trapped beam ions. The term due to passing particles in the growth rate expression may be driving if the width of the passing particle distribution function is bigger then $\delta \lambda_{p}^{2}>1 / 3\left(1-\omega / \omega_{c b}\right)$. One can conclude from the obtained expression that the instability favors low frequency provided the resonance condition is satisfied. Typically the distribution function of beam ions becomes isotropic below the critical energy so that the drive is possible within the energy range $E_{b *}<E<E_{b 0}$ or in NSTX $20 k e V<E<80 k e V$, i.e. $v_{b 0} / 2<v<v_{b 0}$. Trapped particles can be in a resonance if their parallel velocity satisfies $v_{\|} / v_{A}>\left(1+\omega_{c b} / \omega\right)$ for GAE's and $v_{\|} / v_{A}>k_{\perp}\left(1+\omega_{c b} / \omega\right) / k_{\|}$for CAEs.

\section{Thermal ion stochastic heating due to high frequency Alfvén Eigenmodes}

A very important aspect of CAE instability in STs is their nonlinear evolution and saturation. CAEs are considered as candidates to explain the high thermal ion temperature in NSTX through stochastic heating mechanism [15]. Experimentally more evidence of the correlation of the CAE instability with the increase in the background ion temperature is needed to confirm this idea [2]. We simulated this effect in more complex toroidal geometry (see Fig. 4) than was reported previously[15]. Stochastic domain at low energy corresponding to the plasma background ions occurs at lower amplitude than in the slab plasma. Initial temperature profile of thermal ions is shown as a solid parabolic curve in Fig.4. We used 21 CAEs to simulate measured CAE spectrum by edge Mirnov probes in NSTX. Internal measurements of rms amplitude of the perturbations is not available and was fixed $\delta B / B=0.5 \times 10^{-3}$ in calculations with the collisional frequency $\nu=0.01 \omega_{c}$. Mode frequencies were in the range $0.2<\omega / \omega_{c b}\left(R=R_{a x}+a\right)<0.8$. Calculations indicate that the heating is proportional to the mode numbers if the amplitude is fixed. Stochastic multimode 
heating by CAEs could provide a way of direct energy channeling from beam ions to the thermal plasma ions.

\section{Conclusions}

High frequency modes with frequencies below the fundamental cyclotron frequency of thermal ions were observed in NSTX. Based on the measured spectrum of high frequency modes we identified them as CAEs and GAE's. CAE frequency peaks have similar time evolution as plasma parameters change, while GAE peaks may intersect due to $q$-profile relaxation. Theory is presented to study properties of these modes. Both types of instabilities are driven by the NBI tangential injection in NSTX. Beam ions excite CAE/GAE's through the Doppler shifted cyclotron resonance. The main source for the drive is the velocity space anisotropy of the beam ion distribution function. One of the major damping mechanisms of GAE's is the continuum damping and is derived and shown to be small for high poloidal mode numbers and non-flat Alfvén continuum near the center.

Our simulation of the effect CAE/GAE's may have on plasma ions indicate that these modes may provide a channel for efficient energy transfer from NBI directly to thermal ions.

\section{References}

1. FREDRICKSON, E., et. al., Phys. Rev. Lett. 87 (2001) 145001.

2. FREDRICKSON, E., et. al., Phys. Plasmas 9 (2002) 2069.

3. GORELENKOV, et. al., Nucl.Fusion 42 (2002) 977.

4. GORELEnKOV, et. al., Phys. Plasmas 9 (2002) 3483.

5. APPERT, K., et. al., Plasma Physics 24 (1982) 1147.

6. BELOVA E. V. et al., Phys. Plasmas 7 (2000) 4996.

7. BELOVA E. V., et.al., "Numerical simulations of CAE modes in NSTX", Bull. Am. Phys. Soc. v. 46, 334 (2001) 
8. CHEnG, C. Z. , Phys. Reports 211 (1992) 1.

9. MAHAJAN, S. M., ROSs, D. W., CHEN, G.-L., Phys. Fluids 26 (1983) 2195.

10. Kuvshinov, B. N., Mikhailovskit, A. B., Sov. J. Plasma Phys. 18 (1992) 495.

11. VAN DAM, J.W., et. al., Fusion Technology 18 (1990) 461.

12. BERK, H. L., VAN DAM, J. W., GUO, Z., LINDBERG, D. M., Phys. Fluids B 4 (1992) 1806.

13. ROSENBLUTH, M. N., BERK, H. L., VAN DAM, J. W., Z., LINDBERG, D. M., Phys. Fluids B 4 (1992) 2189.

14. GORELenkOV, N. N., and CHENG, C. Z., Phys. Plasmas 2 (1995) 1961.

15. GATES, D. A., et. al., Phys. Rev. Lett. 87 (2001) 205003.

16. GORELEnKOV, N. N., and CHENG, C. Z., Nucl. Fusion 35 (1995) 1743. 


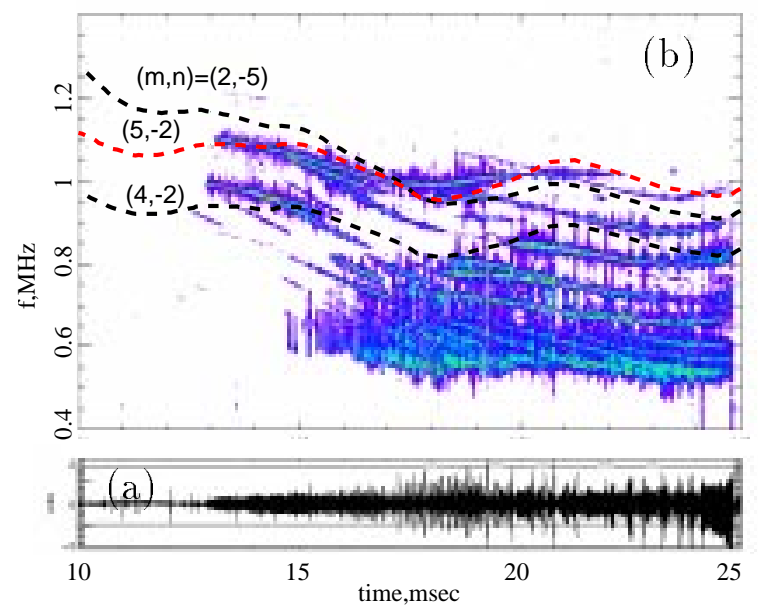

Figure 1: Time evolution of the Mirnov signal (a), and its frequency spectrum (b) in NSTX shot \#108236. 


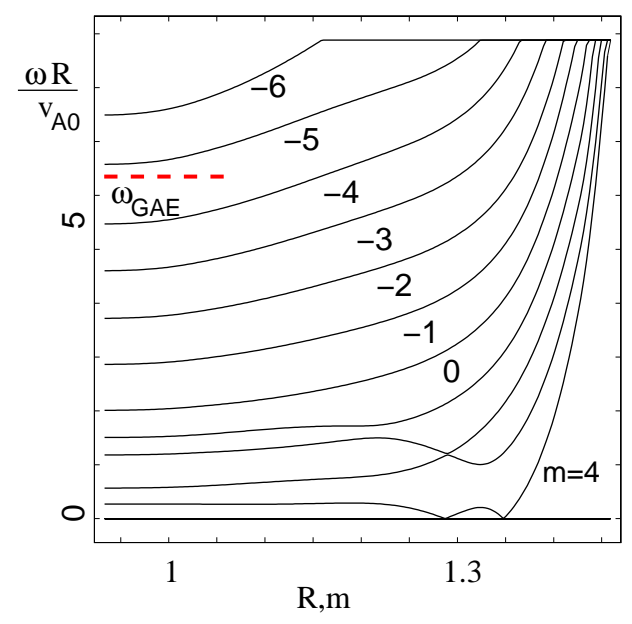

Figure 2: NOVA calculated Alfvén continuum and GAE eigenfrequency for NSTX shot $\# 108236$ at $t=15 \mathrm{msec}$ and for $n=2$. Included are poloidal harmonics from $m=-6$ to $m=4$. Shown as a dashed line is the radial extent and the frequency of GAE with $(m, n)=(-5,2)$. 


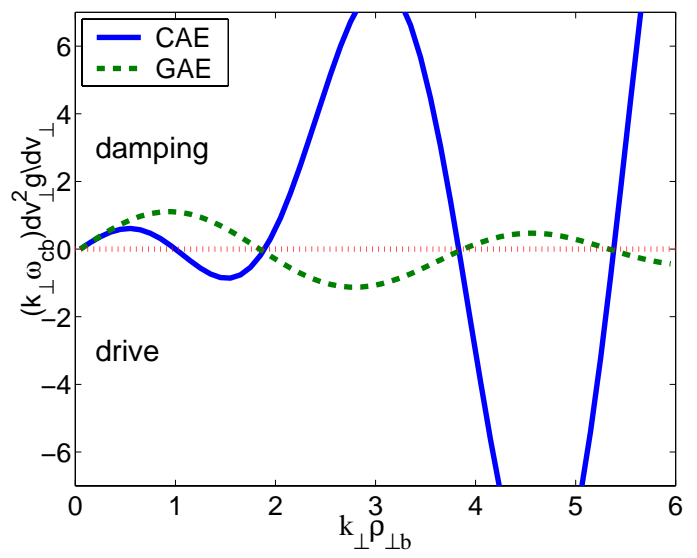

Figure 3: Driving factor from Eq.(17) which includes function $g$ vs. the FLR parameter $k_{\perp} \rho_{\perp b}$. 


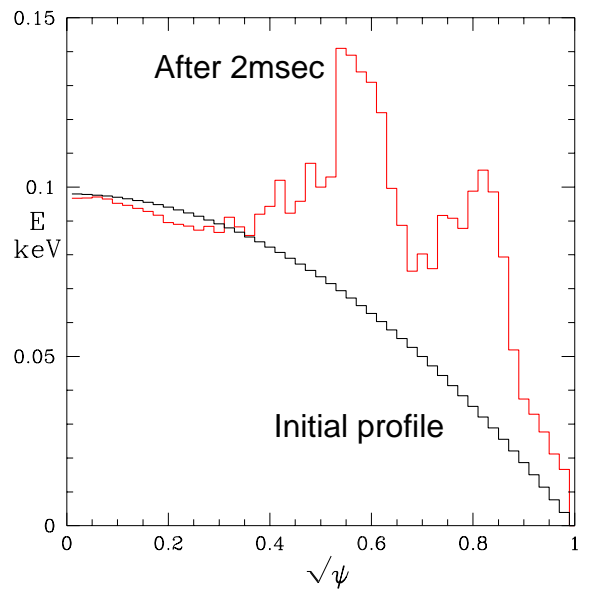

Figure 4: Modeling of thermal ions heating due to multiple CAE modes during $2 \mathrm{msec}$. Also shown is the initial parabolic ion temperature profile. 


\section{External Distribution}

Plasma Research Laboratory, Australian National University, Australia

Professor I.R. Jones, Flinders University, Australia

Professor João Canalle, Instituto de Fisica DEQ/IF - UERJ, Brazil

Mr. Gerson O. Ludwig, Instituto Nacional de Pesquisas, Brazil

Dr. P.H. Sakanaka, Instituto Fisica, Brazil

The Librarian, Culham Laboratory, England

Mrs. S.A. Hutchinson, JET Library, England

Professor M.N. Bussac, Ecole Polytechnique, France

Librarian, Max-Planck-Institut für Plasmaphysik, Germany

Jolan Moldvai, Reports Library, MTA KFKI-ATKI, Hungary

Dr. P. Kaw, Institute for Plasma Research, India

Ms. P.J. Pathak, Librarian, Insitute for Plasma Research, India

Ms. Clelia De Palo, Associazione EURATOM-ENEA, Italy

Dr. G. Grosso, Instituto di Fisica del Plasma, Italy

Librarian, Naka Fusion Research Establishment, JAERI, Japan

Library, Plasma Physics Laboratory, Kyoto University, Japan

Research Information Center, National Institute for Fusion Science, Japan

Dr. O. Mitarai, Kyushu Tokai University, Japan

Dr. Jiangang Li, Institute of Plasma Physics, Chinese Academy of Sciences, People's Republic of China

Professor Yuping Huo, School of Physical Science and Technology, People's Republic of China

Library, Academia Sinica, Institute of Plasma Physics, People's Republic of China

Librarian, Institute of Physics, Chinese Academy of Sciences, People's Republic of China

Dr. S. Mirnov, TRINITI, Troitsk, Russian Federation, Russia

Dr. V.S. Strelkov, Kurchatov Institute, Russian Federation, Russia

Professor Peter Lukac, Katedra Fyziky Plazmy MFF UK, Mlynska dolina F-2, Komenskeho Univerzita, SK-842 15 Bratislava, Slovakia

Dr. G.S. Lee, Korea Basic Science Institute, South Korea

Institute for Plasma Research, University of Maryland, USA

Librarian, Fusion Energy Division, Oak Ridge National Laboratory, USA

Librarian, Institute of Fusion Studies, University of Texas, USA

Librarian, Magnetic Fusion Program, Lawrence Livermore National Laboratory, USA

Library, General Atomics, USA

Plasma Physics Group, Fusion Energy Research Program, University of California at San Diego, USA

Plasma Physics Library, Columbia University, USA

Alkesh Punjabi, Center for Fusion Research and Training, Hampton University, USA

Dr. W.M. Stacey, Fusion Research Center, Georgia Institute of Technology, USA

Dr. John Willis, U.S. Department of Energy, Office of Fusion Energy Sciences, USA

Mr. Paul H. Wright, Indianapolis, Indiana, USA 
The Princeton Plasma Physics Laboratory is operated by Princeton University under contract with the U.S. Department of Energy.

\author{
Information Services \\ Princeton Plasma Physics Laboratory \\ P.O. Box 451 \\ Princeton, NJ 08543
}

Phone: 609-243-2750

Fax: 609-243-2751

e-mail: pppl_info@pppl.gov

Internet Address: http://www.pppl.gov 